\title{
Evaluation of the Equivalence in Dissolution Profiles of Brands of Metronidazole Tablet in Nigeria
}

\author{
Sefunmi Odetola \\ (Clinical Pharmacy and Biopharmacy, College of Pharmacy/University of Lagos, Nigeria)
}

\begin{abstract}
The purpose of this study was to evaluate whether optimum metronidazole preparations are used for the second-line eradication therapy for Helicobacter pylori infection. The regimen uses a combination of antibiotics that includes amoxicillin and metronidazole. Erratic release of metronidazole, leading to sublethal concentration of the drug within the GIT, between different formulations is proposed as a possible reason for acquisition of resistance in H. pylori. A generic brand of metronidazole was compared to the innovator brand, Flagyl. The evaluation was performed by dissolution test and estimation for intragastric drug concentrations was done based on the dissolution data. The dissolution tests were performed using the paddle method of British Pharmacopoeia. The data obtained were used for the evaluation of similarity in intragastric concentrations of metronidazole following drug release. In the study, the time taken to achieve maximum concentration of metronidazole in the simulated gastric fluid, for Flagyl was less than that for the test drug. The reference and test products were found to be similar, both achieving more than $85 \%$ release of drug content in 20minutes in the simulated intestinal fluids. This result implies that the test product can be substituted for Flagyl use in $\mathrm{H}$. pylori eradication therapy in Nigeria.
\end{abstract}

Keywords: dissolution profiles, eradication therapy, helicobacter pylori, in-vitro, metronidazole

\section{Introduction}

Helicobacter pylori, a gram-negative rod, has been associated with gastritis and the subsequent development of gastric and duodenal ulcers, gastric adenocarcinoma, and gastric B-cell lymphoma [1]. Eradication of $\mathrm{H}$. pylori has thus been widely indicated in the treatment of dyspepsia and peptic ulcer disease in adults under the age of 45. Due to antibiotic resistance and a consequent increased risk of incomplete eradication of $\mathrm{H}$. pylori with the first-line eradication regimen that uses clarithromycin, amoxicillin, and a proton pump inhibitor, the second-line eradication therapy was introduced, in which metronidazole is either used in place of clarithromycin or added to the first line antibiotic combinations [2-6]. In the eradication therapy of Helicobacter pylori-associated peptic ulcer, bioavailability of the antibiotic agents used is of great importance to assist in attaining the cure rates desired, while minimizing the risk of resistance development. Furthermore, the emergence of resistance to clarithromycin and metronidazole is increasingly being recognized as an important and possibly the major factor in failure of therapies to eradicate H. pylori[3-7]. Lack of a consistent availability of lethal concentration of metronidazole within the GIT could enhance development of resistance, and could most possibly be associated with the use of drug products containing metronidazole with dissimilar drug release profiles.

The main objective of this study was to establish equivalence in dissolution profile (in terms of amount made available at the site of action of metronidazole in H.pylori-associated peptic ulcer i.e within the gastrointestinal lumen) of a branded metronidazole product to the well established innovator brand Flagyl available in Nigeria. A generic pharmaceutical preparation used in second-line therapy was selected and was tested for a similarity in its drug release profile to that of the innovator brand. An evaluation as to whether the intragastric concentration of metronidazole released from the two preparations was similar was made from the results of dissolution test. To make this comparison of a commonly prescribed generic brand of metronidazole to the reference product, Flagyl, three dosage units per brand were used. The method employed in this study attempts to assess the similarity of dissolution profile of a branded generic metronidazole tablet to that of the reference brand using the dissolution test procedures described in the next section.

As stated above, therapy failure of $\mathrm{H}$. pylori eradication in peptic ulcer patients is associated with a variety of factors amongst which is bioinequivalence of metronidazole tablets used when interchanged. Increased concentration of metronidazole in the gastrointestinal tract, as determined by the amount released from the drug product by dissolution, offers a better response in patients and this must be reproducible in the different formulations used, thus establishing bioequivalence between a commonly prescribed generic and the innovator product is aimed at enhancing effectiveness of therapy where multisource products are used in place of an innovator brand. In addition, microbial resistance to the drug is less likely when bioequivalent products are used to treat infections, other than that of H.pylori, in patients. 


\section{Method}

200mg Flagyl and VG tablets were purchased from pharmacies, pure metronidazole powder was obtained from the School's Central Research Laboratory. Phosphate and acetate buffers were prepared according to the method described in the British Pharmacopoeia[8], using analytical grade reagents supplied by the clinical pharmacy department laboratory. 0.45 -microns micropore filter, syringes and $5 \mathrm{ml}$ sample bottles were purchased from a laboratory reagent and equipments supplier. I gained access to use the Agilent 8453 UV/Visible Spectrophotometer and Dissolution Apparatus II in the School's Central Research Laboratory

The test methods and procedures were carried out as laid out in the pharmacopoeia. A branded generic product code named VG was compared to the reference product, Flagyl. Selection of the generic brand was made based on its availability as at the time of study, and purchased directly from the market. Test media of $\mathrm{pH}$ $1.2,4.5$ and 6.8 were prepared as specified in the BP; the first fluid $(\mathrm{pH} 1.2)$ contains $8.33 \mathrm{mls}$ of $37 \% \mathrm{HCl}$ in 1000 millilitres of distilled water, the second fluid contains 1.92 millilitres of acetic acid and $30 \mathrm{~g}$ of ammonium acetate in 1000 millilitres of distilled water, and the third fluid contains $6.84 \mathrm{~g}$ of potassium diphosphate and $0.92 \mathrm{~g}$ of sodium hydroxide in 1000 millilitres of distilled water. USP dissolution apparatus 2(paddle) was used at a speed of 50rpm; the water bath of the apparatus was maintained at $37^{\circ} \mathrm{C}$. Each 900 millilitres volume of the different media was placed in each vessel of the dissolution apparatus and was allowed to equilibrate for about an hour. Three tablets of each brand were tested in each medium, and each tablet was dropped into the vessel containing the $900 \mathrm{ml}$ medium maintained at $37^{\circ} \mathrm{C}$, and the apparatus was run for about thirty minutes, collecting one milliliter samples at intervals of 5, 10,15,20, and 30 minutes, replacing the samples collected with an equal volume of the freshly prepared medium. Samples collected were filtered through a microfilter of pore size about 0.45 microns, and diluted using a ratio of 1:20. A calibration curve for each medium was prepared using pure metronidazole powder. Serial dilution of stock solutions of pure metronidazole powder with strengths of $4 \mathrm{mg} / \mathrm{ml}, 5.7 \mathrm{mg} / \mathrm{ml}$, and $6 \mathrm{mg} / \mathrm{ml}$ for $\mathrm{pH} 1.24 .5$, and 6.8 media, was made. Absorbance reading of each filtered and diluted sample was taken with an Agilent 8453 model of UV-Visible spectrophotometer, at a wavelength of maximal absorption of $277 \mathrm{~nm}$ for the $\mathrm{pH} 1.2$ medium, and $320 \mathrm{~nm}$ for the $\mathrm{pH} 4.5$ and 6.8 media. Mean concentration of metronidazole released from Flagyl and VG in each medium at intervals of 5, 10, 15, 20 and 30 minutes was obtained from the calibration curve. Percent drug release was obtained from the concentration of metronidazole obtained from the samples collected at each time interval. Mean percent drug release for all three tablets per brand was plotted against time to obtain a dissolution curve that describes the dissolution profile of each brand of metronidazole tablet in the three media. Comparison of dissolution profile of each curve was done to establish bioequivalence between the brands.

\section{Results And Discussion}

The dissolution profiles of Flagyl and VG at pH 1.2 (simulated gastric fluid) are confirmed to be different as shown in the charts below. Fig 1 shows the profile of release of metronidazole from three $200 \mathrm{mg}$ Flagyl and VG tablets in O.IN $\mathrm{HCl}$ over a thirty-minute period.

Release profiles for Flagyl and VG very similar in acetate buffer and phosphate buffers, with Flagyl releasing less, than VG, of its $200 \mathrm{mg}$ content of metronidazole over the thirty minutes period in the acetate buffer. The dissolution characteristics of the two products in $\mathrm{pH} 4.0$ and 6.8 were almost the same. The dissolution rate of Flagyl at $\mathrm{pH} 1.2$ was more rapid than those at $\mathrm{pH} 4.0$ and 6.8. Data at $\mathrm{pH} 1.2$ and 6.8 showed that the time taken for $100 \%$ of the $200 \mathrm{mg}$ label claim of metronidazole to be released from Flagyl tablets were about $16 \mathrm{~min}$ and $15 \mathrm{~min}$. For VG, time taken for $100 \%$ release in $\mathrm{pH} 4.5$ ad 6.8 was about 10 and 15 minutes. The dissolution characteristics of metronidazole from VG and Flagyl in phosphate and acetate buffers were confirmed to be almost identical. Thus, the likelihood of releasing differing concentration of metronidazole in the small intestinal tract, which may be less than that required for effective attack of H. pylori, within the GI lumen is minimal with these two products.

Metronidazole is concentration-dependent antibiotic, and thus its efficacy both in eradicating H. pylori within the intestinal lumen, and in effectively killing other anaerobes at other systemic sites of infection will depend extensively on the rapid release of the drug from the tablet formulation, and also on the dissolution of the drug in the gastric and intestinal media to reach high enough concentration of the solutes, which could then act locally, or be absorbed.

With the results of this study, nearly identical dissolution profiles obtained for the two brands in the phosphate and acetate buffer implies that substitution of either for use in eradication regimen is most probably not to be expected to show difference in in-vivo performance. However, dissolution profile dissimilarity in the simulated gastric media could raise a caution for substitution of the use of the generic (VG) to the innovator brand (Flagyl), in eradication regimens for a predominantly gastric ulcer. Failure of this formulation to release the same concentration of metronidazole within the gastric region over time could also aid resistance development, as the concentration of metronidazole available to attack the colonies of $\mathrm{H}$. pylori in the gastric 
antrum will be sublethal. Since a selection of generic brand was done based on its availability at time of study, generalised statements should be made with a level of caution, from the results of this study.

In vivo performance of metronidazole release from its solid oral dosage formulations generally show a good correlation with in vitro performance, thus allowing for estimations of in vivo performance to be made from in vitro data.

Concentration in $\mathrm{mcg} / \mathrm{ml}$ of metronidazole in the samples was obtained by extrapolation from the calibration curve.

Amount of drug released in $\mathrm{mg} / \mathrm{ml}$ was obtained from the relationship:

Concentration in $\mathrm{mcg} / \mathrm{ml} \times 900 \times$ dilution factor

$$
1000
$$

Percent drug release was expressed as a fraction of $200 \mathrm{mg}$, the label claim of the tablet strength.

Table 1: shows amount of drug released from Flagyl per time interval;

\begin{tabular}{|l|l|l|l|}
\hline Time (minutes) & $\begin{array}{l}\mathrm{pH} 1.2 \\
\text { Mean } \pm \mathrm{SEM}(\mathrm{mg})\end{array}$ & $\begin{array}{l}\mathrm{pH} 4.5 \\
\text { Mean } \pm \mathrm{SEM}(\mathrm{mg})\end{array}$ & $\begin{array}{l}\mathrm{pH} 6.8 \\
\text { Mean } \pm \mathrm{SEM}(\mathrm{mg})\end{array}$ \\
\hline 5 & $162.40 \pm 47.01$ & $137.64 \pm 2.442$ & $58.099 \pm 50.4$ \\
\hline 10 & $150.77 \pm 10.85$ & $164.10 \pm 4.74$ & $175.68 \pm 20.39$ \\
\hline 15 & $155.91 \pm 25.88$ & $196.26 \pm 15.17$ & $199.01 \pm 14.15$ \\
\hline 20 & $287.50 \pm 64.40$ & $186.80 \pm 4.41$ & $190.93 \pm 21.75$ \\
\hline 30 & $60.87 \pm 77.40$ & $190.87 \pm 1.83$ & $263.38 \pm 69.80$ \\
\hline
\end{tabular}

Table 2: shows the amount of metronidazole released from VG per time interval;

\begin{tabular}{|l|l|l|l|}
\hline Time (minutes) & $\begin{array}{l}\mathrm{pH} 1.2 \\
\text { Mean } \pm \text { SEM }(\mathrm{mg})\end{array}$ & $\begin{array}{l}\mathrm{pH} 4.5 \\
\text { Mean } \pm \text { SEM }(\mathrm{mg})\end{array}$ & $\begin{array}{l}\mathrm{pH} 6.8 \\
\text { Mean } \pm \text { SEM }\end{array}$ \\
\hline 5 & $147.17 \pm 7.78$ & $125.58 \pm 6.27$ & $20.69 \pm 6.37$ \\
\hline 10 & $139.75 \pm 1.87$ & $179.30 \pm 6.27$ & $108.54 \pm 14.14$ \\
\hline 15 & $149.50 \pm 0.98$ & $228.07 \pm 54.25$ & $181.02 \pm 17.02$ \\
\hline 20 & $150.07 \pm 21.14$ & $230.70 \pm 65.48$ & $171.02 \pm 17.45$ \\
\hline 30 & $126.57 \pm 0.99$ & $243.84 \pm 60.47$ & $176.83 \pm 2.27$ \\
\hline
\end{tabular}

\subsection{4 : Dissolution Profile Results:}

Figure 1: shows the profile of release of metronidazole from three 200mg Flagyl and VG tablets inO.IN $\mathrm{HCl}$ over a thirty minute period.

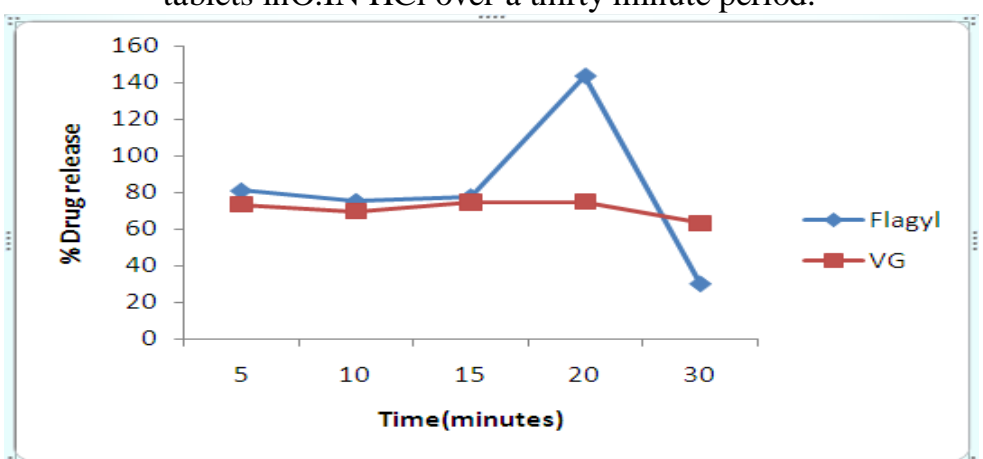

Figure 1: Release profile of Flagyl and VG in simulated gastric fluid over a thirty minutes period.

Figure 2: shows the profile of release of metronidazole from Flagyl and VG in $\mathrm{pH} 4.5$ medium.

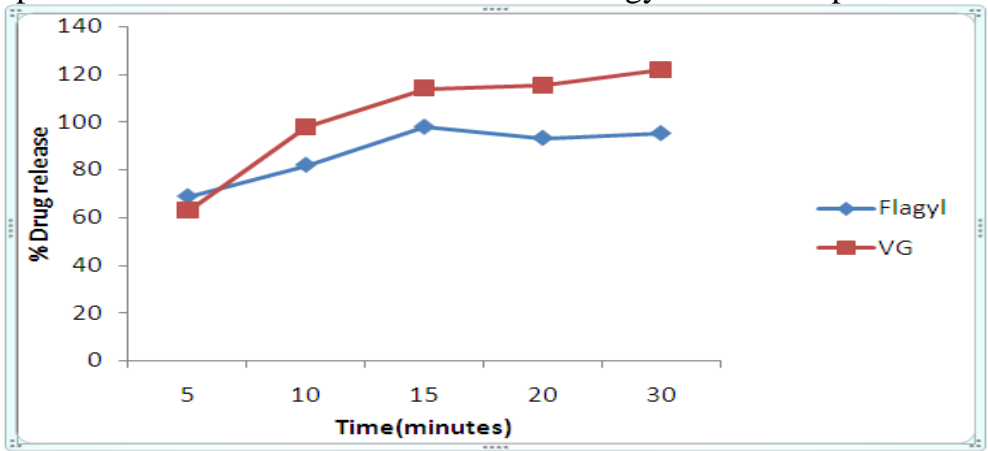

Figure 2: Release profile of metronidazole from 200mg Flagyl and VG tablets in phosphate buffer over a thirty minute period 
Figure 3: shows the profile of release of metronidazole from Flagyl and VG in pH 6.8 medium.

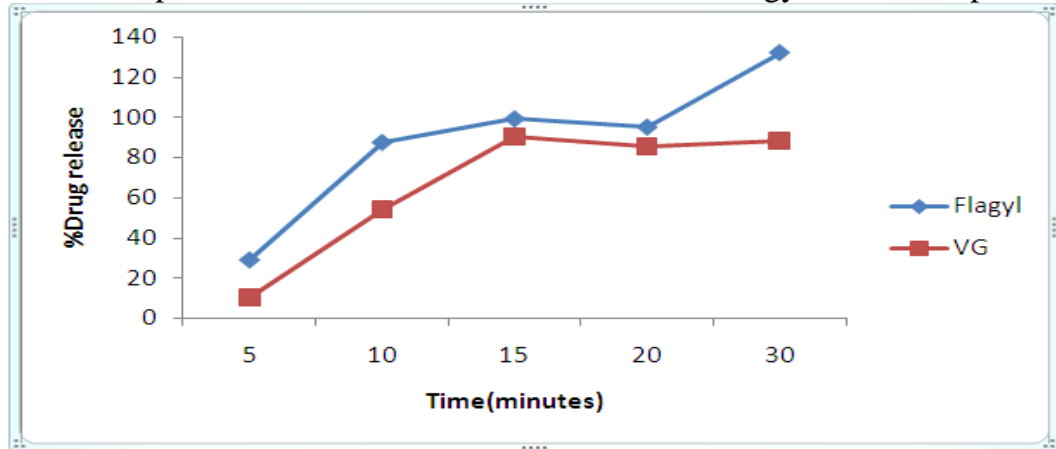

Figure 3: Release profiles for Flagyl and VG in phosphate buffer over a thirty minutes period.

\section{Conclusion}

The findings of this study show the similarity in release profile of metronidazole from the branded and generic product. Release profiles were nearly identical for the two products in simulated intestinal fluids, thus no difference in in-vivo drug release performance is expected when the two products are used for eradication of H. pylori in duodenal ulcer cases under different clinical circumstances. The two products pass the USP recommendation of not less than $85 \%$ of the drug being released in the first 15 minutes, in the simulated intestinal fluids. These results imply that substitution of VG can be made for Flagyl, in clinical use, without placing the patient at a risk of treatment failure that could arise from bioinequivalence of the two drug products. It could also be inferred with caution, that the drug will be made bioavailable from the tablets other extraintestinal sites of action. A difference in profile was observed in the simulated gastric fluid, and caution should be taken in substitution for use in eradication regimens of $\mathrm{H}$. pylori in gastric ulcer cases. The findings of this study also suggests that the test and reference products are bioequivalent, but further studies might need to be done using more tablets to confirm this suggestion.

\section{REFERENCES}

[1]. P Malfertheiner, F Megraud, C O’Morain, F Bazzoli, E El-Omar, D Graham, R Hunt, T Rokkas, N Vakil, and E J Kuipers. Current concepts in the management of Helicobacter pylori infection: the Maastricht III Consensus Report, Gut 56(6), 2007, 772-781.

[2]. T Fujioka, A Yoshikawa, T Okimoto, M Kodama, K Murakami. Guidelines for the management of Helicobacter pylori infection in Japan: current status and future prospects. Journal of Gastroenterology, 42(17), 2007, 3-6.

[3]. DY Graham, L Fischbach. Helicobacter pylori treatment in the era of increasing antibiotic resistance. Gut, 59(8), 2010, 1143-1153

[4]. JP Gisbert, JM Pajares. Evolution of Helicobacter pylori therapy from a meta-analytical perspective. Helicobacter, 12(Suppl 2), 2007, 50-58

[5]. F Megraud. H pylori antibiotic resistance: prevalence, importance, and advances in testing. Gut. 53(9), 2004, 1374-1384

[6]. V De Francesco, F Giorgio, C Hassan, G Manes, L Vannella, C Panella, E Ierardi, A Zullo. Worldwide H. pylori antibiotic resistance: a systematic review. J Gastrintestin Liver Dis, 19(4), 2010, 409-414

[7]. $\quad$ S.C. Sweetman. 2009. Martindale: The complete drug reference. (Pharmaceutical Press 36th ed. London) pp 1703-1704.

[8]. British Pharmacopoeia, 1998 Edition, p. 1367-1369. 